\title{
Prevalence of hypersexual behavior in Parkinson's disease patients: Not restricted to males and dopamine agonist use
}

\author{
Christine A Cooper \\ Armon Jadidian \\ Michelle Paggi \\ Janet Romrell \\ Michael S Okun \\ Ramon L Rodriguez \\ Hubert H Fernandez \\ Department of Neurology, University \\ of Florida, Gainesville, FL, USA
}

\begin{abstract}
This study investigates the prevalence and demographic characteristics of hypersexuality in Parkinson's disease (PD). Impulse control disorders in PD patients have been associated with dopamine agonist therapy. Moreover, hypersexuality and pathological gambling have been associated with males, while females may be inherently thought to be more likely to participate in compulsive shopping and binge-eating behaviors. In this study, a screening mail-in survey was sent to all PD patients at a single Movement Disorders Center. One hundred forty one of $400(35.3 \%)$ research packets were returned completed. Fifteen of 141 patients met initial screening criteria for hypersexual behavior. After detailed interview, only 6/141 (4.3\%) of PD patients met criteria for pathologic hypersexual behavior. These behaviors included: compulsive masturbation, prostitution, and paraphilias. Patients with a younger age of PD onset were more likely to exhibit hypersexual behavior. Unlike previous report, no significant association was found between hypersexuality and gender or dopamine agonist use. Rather, this study suggests that physicians should be vigilant for hypersexual behavior in all PD patients, regardless of gender and PD medication regimen. Ultimately, given the innate sensitivity of the topic and survey limitations, it is very likely that hypersexual behavior in our cohort, as it is in the general PD population, has been under-reported.
\end{abstract}

Keywords: Parkinson's disease, hypersexuality, impulsive behavior, dopamine agonists

\section{Introduction}

Impulse control disorders are increasingly reported in Parkinson's disease (PD). Examples of these iatrogenic behaviors seen in PD include: binge eating, excessive spending, compulsive shopping, pathological gambling, and hypersexuality. ${ }^{1}$ Hypersexuality in particular is a psychiatric issue that patients are often reluctant to discuss. Voon and colleagues found the lifetime prevalence of pathologic hypersexuality in PD to be $2.4 \% .{ }^{1}$ Hypersexual behavior typically entails an increase in premorbid sexual activities as well as an increase in the variety of sexual behaviors. ${ }^{2}$ While no direct cause has been identified, Voon and colleagues found hypersexuality to be significantly associated with male sex and an earlier age of PD onset. ${ }^{1}$ Treatment with dopamine agonist therapy and a history of impulse control disorders has also been proposed as possible risk factors. ${ }^{3}$

This study investigates prevalence and demographic characteristics of hypersexual behavior in PD patients residing in north central Florida, USA.

\section{Methods}

The study was approved by the Institutional Review Board of the University of Florida. Four hundred packets were mailed to all patients diagnosed with idiopathic PD enrolled in the Movement Disorders Center of the University of Florida. The diagnosis was made 
by a fellowship trained movement disorders specialists and was based on fulfilling strict United Kingdom Brain Bank Criteria. ${ }^{4}$ As approved by the University of Florida Institutional Review Board, in the survey phase of this study, the subject's response to the questionnaire was considered an "implied consent". In addition, all patients signed a separate informed consent to allow us to contact them, if needed, and to have their data analyzed. Information on demographics, health history, current therapies, and hypersexual behavior was obtained in addition to the Neuropsychological Inventory (NPI) and Beck's Depression Inventory (BDI).

Patients were asked directly if they participated in hypersexual behavior on the hypersexual behavior questionnaire (see Table 1). As part of the mail-in survey, the patients were asked if they would like to be further contacted in regard to this research survey. Only those patients who consented were subsequently interviewed for confirmation and classification to determine if they met the diagnostic criteria for subsybdromal or pathological hypersexuality as outlined by Voon and colleagues (see Table 2 ). ${ }^{1}$ The patient's significant other participated in the interview if the patient consented and was available at the time.

Continuous demographic variables (eg, age of onset, PD duration, NPI, BDI) were analyzed using unpaired t tests. Categorical demographic variables (eg, gender, handedness, marital status, times married, side of PD onset, smoking history, psychiatric history, family psychiatric history, dopamine agonist therapy, and levodopa therapy) were analyzed using Fisher's exact tests and Chi-squared tests. All statistical analyses were performed using GraphPad Prism (GraphPad Software, Inc., La Jolla, CA, USA) software.

\section{Results}

One hundred forty-one from 400 (35.3\%) patients returned completed research packets. This sample had a mean age of 68.0 years ( \pm 10.2 yrs) and an average PD duration of 6.7 years ( $\pm 5.9 \mathrm{yrs}$ ). Fifteen of 141 respondents self-identified as possessing hypersexual behavior. Only six of 15 patients met criteria for pathological hypersexuality $(n=5)$ or subsyndromal hypersexuality $(n=1)$. Whenever there was a question on the veracity of patient reports or reliability of their testimony, the subject's partner participated in the interviews, and in all cases these patients qualified as exhibiting pathological hypersexual behavior. The prevalence therefore of hypersexuality in our cohort was $4.3 \%(6 / 141)$. In these six patients, the most common hypersexual behavior reported was increased pornography interest (magazine, video, or Internet) (three of the six patients), and compulsive masturbation (two patients).
Individually, patients also reported novel interests in a wide range of behaviors including sexual toys, sadomasochism, prostitution, and cross-dressing. The average age of PD onset was significantly lower in the hypersexual group (50.2 yrs \pm 3.0 ) compared to the nonhypersexual group (61.5 yrs \pm 1.0$)$ $(\mathrm{t}(124)=2.437 ; \mathrm{p}<0.05)$. No significant association was found between hypersexual behavior and gender, handedness, marital status, times married, side of PD onset, psychiatric history, family psychiatric history, dopamine agonist therapy, levodopa therapy, PD duration, NPI or BDI score. Interestingly, this sample showed a significant association between a history of smoking and not developing hypersexual behavior (Fisher's exact test $\mathrm{p}<0.05$ ).

\section{Discussion}

Given the innate sensitivity of the topic, it is very likely that hypersexual behavior is under-reported. Despite the limitations (and probable underestimation) of a mail-in survey, the prevalence of hypersexuality in our PD cohort (4.3\%) was slightly higher than the $2.4 \%$ reported by Voon and colleagues. ${ }^{1}$ Patients with a younger age of PD onset were more likely to exhibit hypersexual behavior.

Previously, hypersexuality was associated with males but not females. ${ }^{1}$ Our current study failed to substantiate this finding. This highlights the possibility of hypersexual behavior in female patients that may be missed due to the inherently small sample size of hypersexual patients.

Although the association between younger age of onset and hypersexual behavior might be thought to be due to a greater use of dopamine agonist therapy in these patients, no significant relationship was found between dopamine agonist therapy and hypersexual behavior in this sample. Klos and colleagues found that hypersexual behavior typically began after eight months of dopamine therapy and then resolved after discontinuing dopamine agonist therapy. ${ }^{5}$ It should be noted that a few patients in our study did mention a similar experience. Shaw and colleagues proposed dopamine therapy in general may cause hypersexuality both directly, by increasing libido and arousal, and indirectly by interacting with reward-center neural substrates. ${ }^{2}$ This is in line with the Voon and colleagues finding that a higher total levodopa dose equivalent, but not dopamine agonist dose, was associated with hypersexuality. ${ }^{1}$ Intuitively, it seems very likely that manipulation of the dopamine system would result in changes in reward-seeking behavior. Additional compulsive behaviors coincide with hypersexuality in up to $60 \%$ of hypersexual PD patients. $^{5}$

Given that only a relatively small percentage of PD patients exhibit hypersexuality or any type of impulse 
Table I Questionnaire: Hypersexual behavior in Parkinson's disease

The following questions address hypersexual behaviors. Please answer the following questions honestly and to the best of your ability. (Please circle your answer)

I. Yes or No Do you participate in hypersexual behaviors? If yes, please answer all questions. If no, please proceed to next questionnaire.

2. Yes or No Do you engage in compulsive sexual behavior?

3. Yes or No Do you exhibit sexual behavior more than you should?

4. How often do you engage in hypersexual behavior? Circle one:
Quarterly
Monthly
Weekly
Daily

5. Yes or No Did you have hypersexual behavior before your diagnosis?

6. Yes or No Has your hypersexual behavior increased since your diagnosis?

7. Yes or No Have you noticed an increase in hypersexual behavior since a medication adjustment? If yes, please describe:

8. What forms of hypersexual behavior do you participate in? You may mark more than one answer.

Compulsive masturbation

Prostitution

Porn mags

Extramarital affairs

Porn videos

Internet porn

Internet sex chat rooms

Heterosexual promiscuity

Homosexual promiscuity

Telephone sex dependence

Use of sexual accessories/or toys

Voyeurism

Other (please describe)

9. Yes or No Is there a history of hypersexual behavior in your family?

10. Yes or No Do you have an increased urge to engage in hypersexual behavior?

II. Yes or No Does your hypersexual behavior bother you or make you feel guilty?

12. Yes or No Has anyone ever said anything to you about your hypersexual behavior?

13. Yes or No Do you find yourself exhibiting hypersexual behavior predominantly either "On" or "Off" medication? If yes, please specify: On Off On and Off

14. Yes or No Do you constantly have sexual thoughts?

15. How do you feel when you are exhibiting hypersexual behavior? You may select more than one answer.

$\begin{array}{llllll}\text { Nervous } & \text { Anxious } & \text { Excited } & \text { Happy } & \text { Relaxed } & \text { Sad } \\ \text { Depressed } & \text { Invincible } & \text { Guilty } & & & \\ \text { Other: } & & & & \end{array}$

16. How do you feel when you are not exhibiting hypersexual behavior? You may select more than one answer.

$\begin{array}{llllll}\text { Nervous } & \text { Anxious } & \text { Excited } & \text { Happy } & \text { Relaxed } & \text { Sad } \\ \text { Depressed } & \text { Invincible } & \text { Guilty } & & & \end{array}$

Other:

17. How do you feel after the hypersexual behavior has been exhibited? You may select more than one answer.

$\begin{array}{llllll}\text { Nervous } & \text { Anxious } & \text { Excited Happy } & \text { Relaxed } & \text { Sad } & \text { Worried } \\ \text { Depressed } & \text { Invincible } & \text { Guilty } & & \\ \text { Other: } & & & & \end{array}$

18. Yes or No Have you ever borrowed money or sold anything to finance your hypersexual behavior? 
Table 2 Proposed operational diagnostic criteria for pathologic hypersexuality'

A. The sexual thoughts or behaviors are excessive or an atypical change from baseline marked by one or more of the following:

I. Maladaptive preoccupation with sexual thoughts.

2. Inappropriately or excessively requesting sex from spouse or partner.

3. Habitual promiscuity.

4. Compulsive masturbation.

5. Telephone sex lines or pornography.

6. Paraphilias.

B. The behavior must have persisted for at least one month.

C.The behavior causes at least one or more of the following:

I. Marked distress.

2. Attempts to control thoughts or behavior unsuccessful or result in marked anxiety or distress.

3. Are time consuming.

4. Interfere significantly with social or occupational functioning.

D. The behavior does not occur exclusively during periods of hypomania or mania.

E. If all criteria except $C$ are fulfilled, the disorder is subsyndromal.

control disorder, it seems likely that susceptibility may be due to underlying heritable temperamental traits such as novelty-seeking. ${ }^{6}$ Moreover, Pontone and colleagues found an association between impulse control disorders and depressed mood, disinhibition, irritability, and appetite disturbance. ${ }^{7}$ Table 3 shows demographic data from our current hypersexual population. While not statistically significant, five out of six patients had either a personal or family history of previous psychiatric disorders. Notably, none of the patients had a history of hypersexual behavior prior to PD onset. Also, there appears to be a trend of elevated depression scores, as measured by the BDI, in a majority of these patients.

It should be noted that this study relied on a population of patients all from a single Movement Disorders Center. Therefore it is possible that it does not represent the general PD population. Similar studies in subsequent populations would help to eliminate the possibility of a population bias.

Also, due to survey limitations, as well as possible patient cognitive decline, it is possible that not all patients understood the survey questions as intended. Since we relied on initial self-identification of hypersexual behavior before interviewing, it is quite possible that not all patients were able or willing to make this identification. In the future, studies with a larger hypersexual sample size might serve to verify significant demographic variables.

Our study suggests that hypersexuality may be more prevalent than currently realized. It confirms previous reports that it occurs more commonly in the younger PD age group. Clinicians should be aware of possible hypersexual behavior in both sexes, regardless of dopamine agonist therapy, and be prepared to provide information and support to their patients and their patients' caregivers.

Table 3 Demographic data of hypersexual behavior patient sample

\begin{tabular}{|c|c|c|c|c|c|c|}
\hline Patient & I & 2 & 3 & 4 & 5 & Subsyndromal \\
\hline Current age & 54 & 64 & 59 & 74 & 52 & 60 \\
\hline Gender & Male & Male & Male & Female & Male & Male \\
\hline Marital status & Married & Married & Married & Married & Married & Married \\
\hline Times married & I & I & I & 4 & 1 & I \\
\hline PD duration (years) & & 19 & I & 25 & 9 & 4 \\
\hline Age of onset & & 45 & 58 & 49 & 43 & 56 \\
\hline Handedness & Right & Right & Left & Right & Right & Right \\
\hline PD side started & Right & Right & Both & Right & Right & Left \\
\hline Alcohol consumption & Never & Never & Occasionally & Never & Never & Never \\
\hline Levodopa & Yes & Yes & No & Yes & Yes & No \\
\hline Dopamine agonist & No & Yes & Yes & Yes & Yes & Yes \\
\hline Past psych history & Yes & No & No & Yes & Yes & Yes \\
\hline Past family psych history & Yes & Yes & No & Yes & No & Yes \\
\hline $\begin{array}{l}\text { Past hypersexual } \\
\text { behavior }\end{array}$ & No & No & No & No & No & No \\
\hline NPI & 10 & 10 & 3 & & 0 & 8 \\
\hline BDI & 18 & 17 & 11 & & 1 & 17 \\
\hline
\end{tabular}

Abbreviations: BDI, Beck's Depression Inventory; NPI, Neuropsychological Inventory; PD, Parkinson's disease. 


\section{Acknowledgments}

This study was funded in part by a National Parkinson's Foundation Center of Excellence Research Grant as well as a summer research grant funded by the Medical Sciences Research Program at the University of Florida College of Medicine.

\section{References}

1. Voon V, Hassan K, Zurowki M, et al. Prevalence of repetitive and reward-seeking behaviors in Parkinson disease. Neurology. 2006;67:1254-1257.

2. Shaw P, Blockley A, Clough C, Chaudhuri R, Weeks R, David AS. Hypersexuality and Parkinson's disease. J Neurol Neurosurg Psychiatry. 2003;74.6:834.
3. Weintraub D, Siderowf AD, Potenza MN, et al. Association of dopamine agonist use with impulse control disorders in Parkinson disease. Arch Neurol. 2006;63:969-973.

4. Hughes AJ, Ben-Shlomo Y, Daniel SE, Lees AJ. What features improve the accuracy of clinical diagnosis in Parkinson's disease: a clinical pathological study, 1992. Neurology. 2001;57(10 Suppl 3):S34-S38.

5. Klos KJ, Bower JH, Josephs KA, Matsumoto JY, Ahlskog JE. Pathological hypersexuality predominantly linked to adjuvant dopamine agonist therapy in Parkinson's disease and multiple system atrophy. Parkinsonism and Relat Disord. 2005;11:381-386.

6. Voon V, Fox SH. Medication-related impulse control and repetitive behaviors in Parkinson disease. Arch Neurol. 2007;64(8):1089-1096.

7. Pontone G, Williams JR, Bassett SS, Marsh L. Clinical features associated with impulse control disorders in Parkinson disease. Neurology. 2006;10;67(7):1118-1119. 
